\title{
Characterizing Safety: Minimal Control Barrier Functions from Scalar Comparison Systems
}

\author{
Rohit Konda, Aaron D. Ames, and Samuel Coogan
}

\begin{abstract}
Verifying set invariance has classical solutions stemming from the seminal work by Nagumo, and defining sets via a smooth barrier function constraint inequality results in computable flow conditions for guaranteeing set invariance. While a majority of these historic results on set invariance consider flow conditions on the boundary, this paper fully characterizes set invariance through minimal barrier functions by directly appealing to a comparison result to define a flow condition over the entire domain of the system. A considerable benefit of this approach is the removal of regularity assumptions of the barrier function. This paper also outlines necessary and sufficient conditions for a valid differential inequality condition, giving the minimum conditions for this type of approach. We also show when minimal barrier functions are necessary and sufficient for set invariance.
\end{abstract}

Index Terms-Constrained control, Optimization

\section{INTRODUCTION}

I $\mathrm{N}$ the context of dynamical systems, safety has become synonymous with set invariance, the property that state trajectories of a system are contained within a given subset of the state space; e.g., see the textbook [1]. Intuitively, invariance can be established by ensuring that a system's vector field evaluated on the boundary of the candidate invariant set is always sub-tangent to the set so that trajectories cannot escape. The main technical challenge of this approach is in defining an appropriate notion of sub-tangency applicable to general sets and finding conditions that extend over the entire set so they can be used for controller synthesis. Recent work on (control) barrier functions provided conditions for set invariance [2], [3], subject to regularity assumptions on the set. The question this paper addresses is: Are these the strongest possible conditions for set invariance?

The main result of this paper is necessary and sufficient conditions on set invariance that are minimal in that they are the least restrictive conditions needed to ensure set invariance. To obtain this result, we begin considering comparison results for scalar systems which lead to a notion of a minimal solution. This motivates the introduction of a minimal barrier function which leverages a comparison result for scalar systems. Minimal barrier functions are necessary and sufficient for set invariance and, importantly, they do not require the

This research was supported by NSF Awards \# 1544332 and \#1749357. R. Konda is with the School of Electrical and Computer Engineering, Georgia Institute of Technology, Atlanta, GA 30332. rkonda6lgatech. edu

A. D. Ames is with the Dept. of Mechanical and Civil Engineering, California Institute of Technology, Pasadena, CA. ames@caltech.edu

S. Coogan is with the School of Electrical and Computer Engineering and the School of Civil and Environmental Engineering, Georgia Institute of Technology, Atlanta, GA 30332. sam.coogan@gatech.edu regularity conditions imposed by the original formulation of barrier functions. Finally, minimal control barrier functions are introduced wherein state dependent input constraints and controller synthesis are considered.

There is a long and rich history of establishing conditions for set invariance, starting with the seminal work by Nagumo [4] continuing with Bony [5], Brezis [6], and others [7], [8]. The modern literature has predominately focused on extending these classical results to when $\mathcal{S}=\{x: h(x) \geq 0\}$, a subset of the domain $\mathcal{D} \subset \mathbb{R}^{n}$, is defined by a smooth output function $h: \mathcal{D} \rightarrow \mathbb{R}$. The most visible example of this is barrier certificates, which were first introduced to verify safety properties of hybrid systems in [9]. Directly invoking Nagumo's theorem gives the familiar condition : $\frac{\partial h}{\partial x} f(x) \geq 0$ on the boundary of $\mathcal{S}$ implies invariance of $\mathcal{S}$, for the state flow $f$. Extensions of barrier certificates have been plenty, see e.g. [10], [11], but a major assumption is regularity of $h$ : specifically that the gradient $\frac{\partial h}{\partial x}$ on the boundary does not vanish and corresponds to the exterior normal vector of $\mathcal{S}$. This assumption is necessary, as a simple counterexample is given by $h(x)=x^{3}$ and $\dot{x}=-1$. This example is further detailed in Example 1 and also appears in [9].

The alternative approach is to enforce a flow constraint over the entire domain: $\frac{\partial h}{\partial x} f(x) \geq-\phi(h(x)) \forall x \in \mathcal{D}$ for a scalar function $\phi$. From the conception of the Lyapunov-like flow constraint with $\phi \equiv \mathbf{0}$, as in [9], significant work has been undertaken to expand the class of functions $\phi$ sufficient to guarantee invariance of $\mathcal{S}$, e.g. see [12], [13]. With a view towards obtaining tighter conditions for $\phi$, a new form of (control) barrier functions was recently introduced in [3], where $\phi$ is required to be an extended class $\mathcal{K}$ function, i.e., it is strictly increasing and $\phi(0)=0$. Importantly, these conditions are necessary and sufficient for set invariance in the case when $\mathcal{S}$ is compact and 0 is a regular value of $h$.

Yet the question remains: can these assumptions, especially with respect to the regularity of $h$, be relaxed further and still guarantee set invariance? Answering this question is important as it allows for the verification of a larger set of invariance specifications for a given system including significant classes of non-regular sets, such as points, limit cycles, subspaces, etc. This leads to the main contribution of the paper: the largest possible set of functions, $\mu$, in which to lower bound the flow via $\frac{\partial h}{\partial x} f(x) \geq-\mu(h(x)) \quad \forall x \in \mathcal{D}$.

Complete proofs of all results are contained in the extended version ${ }^{1}$. Proofs are presented below for a few main results. The extended version also contains several appendices that

\footnotetext{
${ }^{1}$ The extended version is available at arxiv.org/abs/1908.09323
} 
extend the basic results here to time-varying systems, present stability results using minimal barrier functions, compares to other boundary conditions for invariance in the literature, and presents further details regarding necessary conditions to guarantee the existence of a continuous comparison function.

\section{Minimal Barrier Functions}

We study the system

$$
\dot{x}=f(x)
$$

with state $x \in \mathcal{D}$ where $\mathcal{D} \subseteq \mathbb{R}^{n}$ is assumed to be an open set and $f: \mathcal{D} \rightarrow \mathbb{R}^{n}$ is assumed to be continuous. Under this assumption, for any initial condition $x(0)=x_{0}$, there exists a maximum time interval of existence $I[x(\cdot)]=\left[0, \tau_{\max }[x(\cdot)]\right)$ with $\tau_{\max }[x(\cdot)]>0$ in which the solution $x(t)$ is guaranteed to exist.

The Lie derivative of $h$ along the vector field $f$ is denoted $L_{f} h: \mathcal{D} \rightarrow \mathbb{R}$ and defined by $L_{f} h(x):=\frac{\partial h}{\partial x}(x) f(x)$. We denote standard Euclidean norm by $\|\cdot\|$.

Throughout this paper, we will study invariance of sets defined as $\mathcal{S}=\{x \in \mathcal{D}: h(x) \geq 0\}$ for a continuously differentiable function $h: \mathcal{D} \rightarrow \mathbb{R}$.

A set $\mathcal{S} \subseteq \mathcal{D}$ is positively invariant for (1) if, for any $x_{0} \in$ $\mathcal{S}$, all solutions $x(t)$ with $x(0)=x_{0}$ satisfy $x(t) \in \mathcal{S}$ for all $t \in I[x(\cdot)]$.

By studying sets $\mathcal{S}$ defined by inequality constraints of a smooth function $h$, we can develop Lyapunov-like conditions on the time evolution of the scalar value $h$ over the whole domain $\mathcal{D}$. In particular, we observe that if $h(x(t)) \geq 0$ can be assured for all $t \in I[x(\cdot)]$ and for all initial conditions $x_{0} \in \mathcal{S}$, then $\mathcal{S}$ is positively invariant. In contrast, previous results on barrier functions (for example, [9]) focus on extending Nagumo's Theorem, directly verifying that the flow of the system is sub-tangent to the set $\mathcal{S}$.

First we define a minimal solution $\tilde{w}(t)$ defined on $[0, \tau)$ for the scalar initial value problem

$$
\dot{w}=g(w), \quad w(0)=w_{0} \in \mathbb{R},
$$

with $g: \mathbb{R} \rightarrow \mathbb{R}$ a continuous function, as a solution such that, for any other solution $w^{\prime}(t)$ defined on $[0, \tau), \tilde{w}(t) \leq w^{\prime}(t)$ for all $t \in[0, \tau)$.

Minimal solutions will be fundamental in characterizing differential inequalities for systems that do not necessarily have unique solutions. Relaxing uniqueness assumptions is critical, as even systems and barrier functions defined by polynomials can induce nonunique solutions in the differential inequality, as seen in Example 1. With this, we recall the following differential inequality.

Proposition 1. [14, Thm 6.3] Let $\tilde{w}(t)$ be a minimal solution to the the initial value problem (2) with domain $[0, \tau)$ and $g$ being continuous. If $\eta(t)$ is any differentiable function defined on $[0, \tau)$ such that $\dot{\eta}(t) \geq g(\eta(t))$ for all $t \in[0, \tau)$ and $\eta(0) \geq w_{0}$, then $\eta(t) \geq \tilde{w}(t)$ for all $t \in[0, \tau)$.

Motivated by our interest in using scalar differential equations as barrier functions, we are especially interested in scalar systems for which minimal solutions remain nonnegative when initialized at the origin.
Definition 1. A continuous function $\mu: \mathbb{R} \rightarrow \mathbb{R}$ is a minimal function if the minimal solution $\tilde{w}(t)$ defined on $t \in[0, \tau)$ for the initial value problem $\dot{w}=-\mu(w), w(0)=0$ satisfies $\tilde{w}(t) \geq 0$ for all $t \in[0, \tau)$.

With minimal functions introduced, we can now describe a corresponding barrier function condition.

Definition 2. For the system in (1), a continuously differentiable function $h: \mathcal{D} \rightarrow \mathbb{R}$ is a minimal barrier function (MBF) if there exists a minimal function $\mu$ that satisfies

$$
L_{f} h(x) \geq-\mu(h(x)) \quad \forall x \in \mathcal{D},
$$

where $L_{f} h(x)=\frac{\partial h}{\partial x}(x) f(x)$ denotes the Lie derivative.

The notion of a minimal barrier function allows us to then establish invariance of $\mathcal{S}$.

Theorem 1. Consider the system (1) and a nonempty $\mathcal{S}=$ $\{x \in \mathcal{D}: h(x) \geq 0\}$ for some continuously differentiable $h: \mathcal{D} \rightarrow \mathbb{R}$. If $h$ is a MBF as in Definition 2, then $\mathcal{S}$ is positively invariant.

Let $x(t)$ be a solution defined on $\left[0, \tau_{\max }\right)$ to (1) with any $x(0)=x_{0} \in \mathcal{S}$. Observe that $h(x(0)) \geq 0$. Consider the comparison system $\dot{w}=-\mu(w)$ with $w(0)=0$. We first show that $\tilde{w}(t)$ is defined on $\left[0, \tau_{\max }\right)$ as well. Suppose that $\tilde{w}(t)$ is only defined on $\left[0, \tau^{*}\right)$ for $\tau^{*}<\tau_{\max }$. Since $\mu$ is a minimal function, $\tilde{w}(t) \geq 0$ and $\lim _{t \rightarrow \tau^{*}} \tilde{w}(t)=\infty[15$, Corollary 1.1.2]. By Proposition 1, $h(x(t)) \geq \tilde{w}(t)$ for $t \in$ $\left[0, \tau^{*}\right)$, implying $\lim _{t \rightarrow \tau^{*}} h(x(t))=\infty$ and diverges. Since $x(t) \in \mathcal{D}$ for $t \in\left[0, \tau^{*}\right], h(x(t))$ is a well-defined continuous map from $t \in\left[0, \tau^{*}\right]$ to $\mathbb{R}$. Contradiction ensues, as the image of $\left[0, \tau^{*}\right]$ under the continuous map $h$ is compact and therefore bounded, and the claim is shown. Now, Proposition 1 gives that $h(x(t)) \geq w(t) \geq 0$, implying that $x(t) \in \mathcal{S}$ for all $t \in\left[0, \tau_{\max }\right)$. Therefore $\mathcal{S}$ is positively invariant.

The main theoretical component for Theorem 1 comes directly from the differential inequality in Proposition 1, rather than using the classical argument by Nagumo. In this regard, MBFs highlight the strong connection between set invariance and differential inequalities. Furthermore, if the additional assumption that $\mu(w) \leq 0(<0)$ for $w \leq 0$ holds, then the utility of the MBF is similar to a set-based Lyapunov function for guaranteeing stability (asymptotic stability). A more detailed discussion can be found in the Appendix B of the extended version.

\section{A. Necessary and sufficient conditions for minimal functions}

Relaxing the standard Lyapunov condition $L_{f} h \geq 0$ for all $x \in \mathcal{D}$ has been studied in a number of works, see e.g. [13], [3]. By considering a larger class of comparison functions to lower bound the flow, i.e. $L_{f} h(x) \geq-\phi(h(x))$, it becomes possible to ensure invariance without requiring stability, allowing for a larger design space when constructing valid barrier functions.

We remark that minimal functions, by definition, represent the largest possible class of comparison functions, and in a certain sense, are the most general class of functions that 
can be used in a barrier function condition. However, since minimal functions are implicitly defined through the resulting nonnegative solutions, checking if a function is indeed minimal is not immediately apparent. Thus the next theorem presents verifiable conditions on $\mu$ to ensure that it is a minimal function. It is important to note that since the next theorem is necessary and sufficient, it represents the tightest possible conditions for a minimal function. The crux of the theorem comes from uniqueness results in [16].

Theorem 2. A continuous function $\mu: \mathbb{R} \rightarrow \mathbb{R}$ is a minimal function if and only if one of the following cases is satisfied:

1) $\mu(0)<0$

2) $\mu(0)=0$ and there exists $\varepsilon>0$ such that $\mu(w) \leq 0$ for all $w \in[-\varepsilon, 0)$

3) $\mu(0)=0$ and for every $\varepsilon>0$, there exists some $w^{\prime}, w^{\prime \prime}$ in $[-\varepsilon, 0]$ such that $\mu\left(w^{\prime}\right)>0$ and $\mu\left(w^{\prime \prime}\right)<0$

4) $\mu(0)=0$ and there exists $k>0$ such that for all $\varepsilon$ with $0<\varepsilon<k, \mu(w) \geq 0$ on $[-\varepsilon, 0]$ and $-1 / \mu(w)$ is not integrable on $[-\varepsilon, \overline{0}]$, i.e. $\int_{0}^{-\varepsilon}-\frac{d w}{\mu(w)}$ is divergent

Case 1 and Case 2 are similar in vein to the standard Lyapunov condition, as $L_{f} h \geq-\mu(h) \geq 0$ on $h \in[-\varepsilon, 0]$ for some $\varepsilon>0$. Case 3 considers the case when $\mu$ changes sign infinitely often. Case 4 relaxes the usual locally Lipschitz condition to a one-sided nonintegrability condition to handle a more general class of comparison functions.

Uniqueness functions have also appeared in the literature as a means for establishing invariance [17]-[19]. Essentially, $g$ is a uniqueness function if any continuously differentiable $\eta(t)$ satisfying $\eta(0)=0$ and $\dot{\eta}(t)=g(\eta(t))$ for all $t$ must necessarily be the unique solution $\eta(t) \equiv 0$ [7].

It can be seen that all continuous uniqueness functions are minimal functions, but $\mu(w)=-w^{\frac{2}{3}}$ is an example of a minimal function that is not a uniqueness function. In this way, the definition of minimal functions captures the essential philosophy of barrier functions: invariance is certified by the nonexistence of solutions to the comparison system in (2) that become strictly negative, and nonunique nonnegative solutions are not relevant to establishing invariance.

We also remark that there are other notions of uniqueness functions used in [7], [19] which are of the same form as minimal functions, but are not used in a comparison framework. In this paper, we directly invoke comparison systems induced by minimal functions to establish invariance in the least restrictive sense.

If a minimal function is a priori known to induce unique solutions, then only the condition that $\mu(0) \leq 0$ needs to be checked. More specifically, it can be verified that all locally Lipschitz minimal functions with $\mu(0) \leq 0$ do indeed satisfy the hypotheses of Theorem 2.

Corollary 1. Any locally Lipschitz continuous function $\mu_{L}$ : $\mathbb{R} \rightarrow \mathbb{R}$ with $\mu_{L}(0) \leq 0$ satisfies the hypotheses of Theorem 2 and therefore is a minimal function.

\section{B. Examples}

The following examples and anti-examples demonstrate the utility of the proposed formulation of minimal barrier functions. We begin with an anti-example that highlights the importance of considering minimal solutions to differential inequalities when constructing comparison systems.

Example 1. Consider $\dot{x}=f(x)=-1$ for $x \in \mathbb{R}$ and let $h(x)=x^{3}$. Take $\mu(w)=3\left(w^{1 / 3}\right)^{2}$. Then $L_{f} h(x)=$ $-\mu(h(x))$ for all $x \in \mathbb{R}$.

Although the function $\mu$ satisfies $\mu(0) \leq 0$, it is not locally Lipschitz and Corollary 1 does not apply. Moreover, $\mu$ does not satisfy any of the conditions of Theorem 2 . Indeed, $\mathcal{S}=\mathbb{R}_{\geq 0}$ is not positively invariant on $\mathbb{R}$.

Further, even though the comparison system $\dot{w}=-\mu(w)$ with the initial condition $w(0)=0$ has a solution $w(t) \equiv 0$, it also has the minimal solution $\tilde{w}(t)=-t^{3}$. Considering $x(t)$, the solution to $\dot{x}=f(x)$ with $x(0)=0$, we see that $h(x(t))=\tilde{w}(t)$, i.e., the barrier function $h$ evaluated along solutions of the system $\dot{x}=f(x)$ just match the minimal solution of the comparison system.

The following example examines the case where the set $\mathcal{S}$ has corners, but still can be verified using a minimal barrier function.

Example 2. Consider the system

$$
\begin{aligned}
& \dot{x}_{1}=-a x_{1}+b x_{2} \\
& \dot{x}_{2}=c x_{1}-d x_{2}
\end{aligned}
$$

where $a, b, c, d \geq 0$. Let a barrier function be $h(x)=x_{1} x_{2}$ so that $\mathcal{S}$ is the union of the first and third quadrants of the plane. $L_{f} h(x)=-a x_{1} x_{2}+b x_{2}^{2}+c x_{1}^{2}-d x_{1} x_{2} \geq-a x_{1} x_{2}-d x_{1} x_{2}=$ $(-a-d) h(x)$ so that (3) is satisfied with $\mu(w)=(a+d) w$ and $\mathcal{S}$ is positively invariant.

In the next example, it is necessary to consider a nonLipschitz minimal function to establish forward invariance with a given barrier function. Even though the vector field of the system is Lipschitz, and the barrier function $h$ is smooth, the resulting dynamics for $L_{f} h$, as a function of $h$, may not be Lipschitz.

Example 3. Consider $\dot{x}=-|x|$ for $x \in \mathbb{R}$ and let

$$
h(x)= \begin{cases}\exp (-1 / x) & \text { if } x \geq 0 \\ -\exp (1 / x) & \text { if } x<0\end{cases}
$$

so that $\mathcal{S}=\{x: h(x) \geq 0\}=\mathbb{R}_{\geq 0}$ is indeed invariant. Define a minimal function candidate

$$
\mu(w)= \begin{cases}-w \ln (w) & \text { if } 0 \leq w<1 \\ w \ln (-w) & \text { if }-1<w<0\end{cases}
$$

and observe that $L_{f} h(x)=-\mu(h(x))$. We check that $\mu$ is a minimal function. Indeed, $\mu(h)$ is continuous with $\mu(0)=0$ and $\mu(h)>0$ over $\mathcal{D} \backslash\{0\}$. Corresponding to Case 4 in Theorem 2, we check that the improper integral

$$
\int_{0}^{-a}-1 / \mu(w) d w=-\left.\ln (\|\ln (-w)\|)\right|_{0} ^{-a}
$$

diverges to $\infty$ for any $a \in(0,1)$. Therefore $\mu$ is a valid minimal function. Observe that $\mu$ is not locally Lipschitz at 0 . Indeed, it can be established that there exists no locally 
Lipschitz minimal function satisfying (3) since any such function must be lower bounded by $\mu$ constructed above and be non-positive at the origin. Explicit calculations are shown in the extended version.

\section{Comparing to Zeroing Barrier Functions}

In this section, we compare MBFs to zeroing barrier functions (ZBFs) in [20], which use extended class $\mathcal{K}$ functions for the class of comparison functions, and is a major inspiration for the work in this paper. A function $\alpha: \mathbb{R} \rightarrow \mathbb{R}$ is extended class $\mathcal{K}$ if it is strictly increasing with $\alpha(0)=0$. We remark that if $\alpha$ is an extended class $\mathcal{K}$ function, then $\alpha$ is a minimal function, as guaranteed by Case 2 in Theorem 2 .

In [20], the development of ZBFs requires utilizing Nagumo's Theorem and therefore requires the assumption that $\frac{\partial h}{\partial x}$ does not degenerate to a zero vector on the boundary of the set. By directly invoking a differential inequality, as in Proposition 1, we can dispense with this assumption, which is discussed further in Section III.

However, restricting to extended class $\mathcal{K}$ functions imposes the barrier condition $L_{f} h(x) \geq-\alpha(h(x)) \geq 0$ for all $x \in \mathcal{D}$, even when $h(x) \leq 0$. This requires stability of each level set $\{x \in \mathcal{D}: h(x)=w\}$ for any $w<0$. While this robustness is desirable in some instances, it does not hold for a large class of systems and sets $\mathcal{S}$. For example, considering the simple system $\dot{x}=x$, it can be shown that there does not exist a class $\mathcal{K}$ function $\alpha$ and a ZBF $h(x)$ such that $L_{f} h(x) \geq-\alpha(h(x))$ holds on all of $\mathcal{D}=\mathbb{R}$ to verify that $\mathcal{S}=\mathbb{R}_{\geq 0}$ is invariant (see Example 5 in the extended version for more details).

Moreover, it is not possible to simply restrict the ZBF to be defined only on $\{x \in \mathcal{D}: h(x) \geq 0\}=\mathcal{S}$. Indeed, this contradicts the hypotheses in Theorem 1 that requires (3) to hold for all $x \in \mathcal{D}$, and ignoring this requirement can result in the following scenario.

Example 4. Consider again Example 1, and take

$$
\alpha(w)= \begin{cases}3 w^{2 / 3} & \text { if } w \geq 0 \\ -3 w^{2 / 3} & \text { if } w<0\end{cases}
$$

so that $L_{f} h(x)=-\alpha(h(x))$ for all $x \in \mathcal{S}$, although notably the equality does not hold for $x \in \mathbb{R} \backslash \mathcal{S}$. and thus Theorem 1 is not applicable since it requires (3) to hold for all $x \in \mathcal{D}$. Notice that $\alpha$ is an extended class $\mathcal{K}$ function on $\mathbb{R}$ and that $-\alpha$ is a minimal function. While it is tempting to use $\dot{w}=-\alpha(w)$ as a comparison system with $w(0)=h(x(0))$, we obtain the false conclusion that $\mathcal{S}$ is positively invariant.

\section{DISCUSSION ON REgULARITY}

Arguably, the most common approach for establishing positive invariance of a set $\mathcal{S}$ is to verify, in some appropriate sense, that the velocity field of the system points inwards to $\mathcal{S}$ at each point on the boundary of $\mathcal{S}$. First formalized by Nagumo in [4] and independently discovered by others, there has since been a volume of work dedicated to making this basic approach precise in various contexts, e.g. [19], [1], [12]. We consider the important specialization of Nagumo's
Theorem to the case where $\mathcal{S}=\{x: h(x) \geq 0\}$ for a smooth function $h: \mathcal{D} \rightarrow \mathbb{R}$.

For a continuously differentiable function $h: \mathcal{D} \rightarrow \mathbb{R}$ for an open set $\mathcal{D} \subseteq \mathbb{R}^{n}, \lambda \in \mathbb{R}$ is a regular value of $h$ if $\frac{\partial h}{\partial x}(x) \neq 0$ for all $x \in\{x \in \mathcal{D}: h(x)=\lambda\}$.

We now recall a version of Nagumo's Theorem, vital to the construction of barrier functions in [9], [20], etc.

Proposition 2 ( [1, Sec 4.2.1]). Consider the system (1) under the added condition that solutions are unique, and a nonempty set $\mathcal{S}=\{x \in \mathcal{D}: h(x) \geq 0\}$ for some continuously differentiable $h: \mathcal{D} \rightarrow \mathbb{R}$. Further assume that 0 is a regular value of $h$. Then $\mathcal{S}$ is positively invariant if and only if

$$
L_{f} h(x) \geq 0
$$

for all $x \in\{x \in \mathcal{D}: h(x)=0\}$.

Proposition 2 provides a powerful result for establishing invariance of $\mathcal{S}$ provided that 0 is a regular value of $h$. In this case, we can equivalently state the condition in (10) as

$$
L_{f} h(x) \geq-\phi(h(x)) \quad \forall x \in \mathcal{D}
$$

where $\phi: \mathbb{R} \rightarrow \mathbb{R}$ is any function with $\phi(0) \leq 0$. Notice that 0 being a regular value allows us to discount much of the structure of $\mu$ defined for Theorem 2. However, as shown in the next theorem, under further mild conditions, the existence of a locally Lipschitz comparison function is guaranteed.

Theorem 3. Let $h: \mathcal{D} \rightarrow \mathbb{R}$ be a twice continuously differentiable function, assume $f$ in (1) is locally Lipschitz, and suppose $\Lambda_{\delta}:=\{x \in \mathcal{D}:-\delta \leq h(x) \leq \delta\}$ is compact for all $\delta \geq 0$. Further assume that 0 is a regular value of $h$. Then there exists a locally Lipschitz function $\mu_{L}$ such that $\mu_{L}(0) \leq 0$ and

$$
L_{f} h(x) \geq-\mu_{L}(h(x)) \quad \forall x \in \mathcal{D} .
$$

Ensuring smoothness properties of the comparison function is useful in generating constraint-based controllers, which is further discussed in Section IV.

Moreover, we have the following immediate corollary.

Corollary 2. Given the assumptions in Theorem $3, \mathcal{S}=\{x \in$ $\mathcal{D}: h(x) \geq 0\}$ is positively invariant if and only if $h$ is a minimal barrier function.

Notice that no regularity assumption is made on $h$ in Theorem 1. Removal of this assumption is due to the structural conditions on $\mu$. On the other hand, Corollary 2 shows that essentially any candidate barrier function $h$ with 0 a regular value and $\mathcal{S}$ being compact has a locally Lipschitz comparison function. Therefore we can use Theorem 1 without loss of generality from Proposition 2 for compact sets, and this allows for considering a significant class of sets $\mathcal{S}$ that are not regular. For example, proving invariance of points, cycles, or any other lower dimensional manifold is possible with the theory of minimal barrier functions. In such cases, 0 cannot be a regular value of the barrier function $h$ because the set $\mathcal{S}=\{x: h(x) \geq 0\}$ has measure zero. In addition, Examples 2 and 3 provide other cases that can be considered with MBFs for which 0 is not a regular value. 


\section{Minimal Control Barrier Functions}

A major benefit for using differential inequalities defined over the whole domain rather than just a boundary-type condition is that it is more amenable to controlled invariance. In constraint-based control, it is desirable to have constraints on the controller that are applied at every point on the domain rather than just a condition on the boundary of $\mathcal{S}$. If a boundary-type condition is directly applied for controlled invariance, the constraints are only active on a measure zero set, which may introduce discontinuities in the controller and render it sensitive to model and sensor noise.

Extensions of minimal barrier functions to control formulations is direct. In this section, we instead consider a control affine system of the form

$$
\dot{x}=f(x)+g(x) k(x)
$$

with state $x \in \mathcal{D}$, where $\mathcal{D} \subseteq \mathbb{R}^{n}$ is assumed to be an open set, a feedback controller $k: \mathcal{D} \rightarrow \mathbb{R}^{m}$, and $f: \mathcal{D} \rightarrow \mathbb{R}^{n}$ and $g: \mathcal{D} \rightarrow \mathbb{R}^{n \times m}$ are both assumed to be continuous. We also assume that $k(x) \in U(x)$ for all $x \in \mathcal{D}$, where $U: \mathcal{D} \rightrightarrows \mathbb{R}^{m}$ is a point-to-set map defining state-based input constraints. Point-to-set maps are denoted with $\rightrightarrows$ for ease of notation. Further define $\mathcal{U}$ as the viable set of continuous controllers

$$
\mathcal{U}=\{k \text { continuous : } k(x) \in U(x) \forall x \in \mathcal{D}\} .
$$

In the rest of the section, we assume $U(x)$ defines a set of state-based affine input constraints of the form

$$
U(x)=\left\{u \in \mathbb{R}^{m}: A(x) u \preceq b(x)\right\}
$$

where $A: \mathbb{R}^{n} \rightarrow \mathbb{R}^{k \times m}$ and $b: \mathbb{R}^{n} \rightarrow \mathbb{R}^{k}$ are both assumed to be continuous in $x$ and $A(x)_{i} u \leq b(x)_{i}$ holds elementwise. General convex input constraints with more detailed proofs are treated in the extended version.

A set $\mathcal{S} \subseteq \mathcal{D}$ is positively controlled invariant if there exists a continuous controller $k$ within the possible class of controllers $\mathcal{U}$ such that $\mathcal{S}$ is positively invariant with respect to the closed loop system $\dot{x}=f(x)+g(x) k(x)$ [1, Def 4.4].

We now state the corresponding definition of minimal barrier functions for control affine systems.

Definition 3. For the control affine system in (13), a continuously differentiable function $h: \mathcal{D} \rightarrow \mathbb{R}$ is a minimal control barrier function (MCBF) if there exists a minimal function $\mu$ such that for all $x \in \mathcal{D}$,

$$
\sup _{u \in U(x)}\left[L_{f} h(x)+L_{g} h(x) u\right] \geq-\mu(h(x))
$$

where $L_{f} h(x)=\frac{\partial h}{\partial x} f(x)$ and $L_{g} h(x)=\frac{\partial h}{\partial x} g(x)$ denote corresponding Lie derivatives.

The set of viable controls is described by the point-to-set map $K: \mathcal{D} \rightrightarrows \mathbb{R}^{m}$ given by

$$
K(x)=\left\{u \in U(x): L_{f} h(x)+L_{g} h(x) u \geq-\mu(h(x))\right\} .
$$

Verification of the existence of controllers with certain properties can be treated as a selection problem, which has been extensively studied in topology [21]. Specifically, the feedback controller $k$ is a selection of $K$ if $k(x) \in K(x)$ for all $x \in \mathcal{D}$. Note that for a controller $k$ to render the set $\mathcal{S}$ invariant, it must necessarily be a selection from the point-toset map $K$.

Additionally, the controller $k$ must come from the set of continuous viable controllers $\mathcal{U}$ in order to guarantee existence of solutions for the closed loop system. Furthermore, continuity of $k$ is also necessary to apply the differential inequality in Proposition 1 and to satisfy the proposed definition of positive controlled invariance.

Theorem 4. Given the control affine system (13), consider a nonempty $\mathcal{S}=\{x \in \mathcal{D}: h(x) \geq 0\}$ for some continuously differentiable $h: \mathcal{D} \rightarrow \mathbb{R}$. If $h$ is a MCBF as in Definition 3 and there exists a continuous controller $k \in \mathcal{U}$ such that $k$ is a selection of $K$, then $\mathcal{S}$ is positively controlled invariant.

To guarantee existence of a continuous controller $k, K$ being nonempty is not sufficient, and additional conditions on $K$ must be assumed. The next theorem gives sufficient conditions on the existence of a continuous controller $k$ that is a selection of $K$ and therefore can be used to satisfy Theorem 4 to render $\mathcal{S}$ positively invariant.

With $U$ characterized as in (15), we also denote the strict interior $K_{I}: \mathcal{D} \rightrightarrows \mathbb{R}^{m}$ as

$$
\begin{aligned}
K_{I}(x)=\left\{u \in \mathbb{R}^{m}\right. & : A(x) u \prec b(x) \\
& \left.L_{f} h(x)+L_{g} h(x) u>-\mu(h(x))\right\} .
\end{aligned}
$$

where the input constraints are described with a strict inequality.

Proposition 3. Given $U$ is defined as in (15), if $K_{I}(x)$ as defined in (18) is nonempty for each $x$, then there exists a continuous controller $k$ that is a selection of $K$.

Proposition 3 is based on the well known Michael's selection theorem [21]. Proposition 3 considers the converse direction of Theorem 4, namely what conditions on $h$ are necessary for there to exist a controller to render $\mathcal{S}$ invariant.

Usually, a controller $k$ is selected from $K$ based on some optimality criteria. A common approach for safety based control is to first obtain a nominal controller $k_{n o m}: \mathcal{D} \rightarrow \mathbb{R}^{m}$ that is not verified for either guaranteeing invariance or satisfying input constraints. The nominal controller is then used within a quadratic optimization program (QP) in which $\hat{k}$ is selected from $K$, while minimizing the distance from $k_{n o m}(x)$ at each $x$, that is,

$$
\hat{k}(x)=\underset{u \in K(x)}{\operatorname{argmin}}\left\|u-k_{n o m}(x)\right\|^{2}
$$

Synthesizing controllers in this fashion can allow for realtime control synthesis that satisfies both performance objectives and safety constraints. For a review of applications of this framework, see [20].

Properties of the controller $\hat{k}$ can be analyzed as a selection of $K$, and conditions on $K$ can be formulated to guarantee continuity of $\hat{k}$. In [22] and [23], Lipschitz continuity of controllers for quadratic programs regarding a minimum norm controller of $k_{n o m} \equiv 0$ for all time was explored. In [23], conditions for pointwise continuity were given, but in this paper, we show continuity of the controller over the whole domain. 
The next theorem gives practical conditions on when the quadratic program in (19) gives a continuous controller.

Theorem 5. Given $U$ is defined as in (15) with $\bigcup_{x \in \mathcal{D}} U(x)$ being compact and $k_{n o m}$ is continuous in $x$, if $K_{I}(x)$ as defined in (18) is nonempty for each $x$, the controller $\hat{k}$ defined by the quadratic program in (19) is continuous.

We first claim that $K$ is upper (u.s.c) and lower semicontinuous (l.s.c) and thus a continuous point-to-set map (see section III in the extended version for definitions). Let $K(x)$ be written as the inequality constraint $G(x, u) \leq 0$ with $G(x, u)=\left[A(x) ;-L_{g} h(x)\right] u-\left[b(x) ;-\mu(h(x))-L_{f} h(x)\right]$. As $G$ is continuous in both $x$ and $u, K$ is a closed map [24, Thm 10] and since $K$ is assumed to map into the compact set $\bigcup_{x \in \mathcal{D}} U(x), K$ is u.s.c [25, Sec 6.1 Thm 7]. Furthermore, $K_{I}(x)$ is assumed to be nonempty for each $x$ and $K$ is affine in $u$ for each fixed $x$. Therefore, the closure of $K_{I}(x)=K(x)$ [24] and then $K$ is 1.s.c [24, Thm 13] as well, and the claim is shown.

The induced point-to-set map $k_{n o m}^{\prime}(x)=\left\{k_{n o m}(x)\right\}$ is continuous by assumption of continuity of $k_{n o m}$. Thus, the product map $K \times k_{n o m}^{\prime}$ is continuous [25, Sec 6.4 Thm 4]. Since $K(x) \times k_{\text {nom }}^{\prime}(x) \subset \mathbb{R}^{m} \times \mathbb{R}^{m}$ is convex and compact and $\left\|u-k_{n o m}(x)\right\|^{2}$ is strictly convex in $u$ for a fixed $x$, there is a unique minimizer of $K \times k_{\text {nom }}^{\prime}$ for each $x$. So $\operatorname{argmin}_{u \in K(x)}\left\|u-k_{n o m}(x)\right\|^{2} \times k_{\text {nom }}(x)$ is a continuous function [25, Sec 6.3 Max Thm ]. Projecting down to the first argument preserves continuity, and therefore, by definition, $\hat{k}$ is continuous.

If our viable control set $K(x)$ is compact and strictly feasible everywhere, Theorem 5 ensures that the optimal controller $\hat{k}$ is continuous. Since $\hat{k}(x) \in K(x)$ for all $x \in \mathcal{D}$ as well, $\hat{k}$ is applicable to Theorem 4, guaranteeing $\mathcal{S}$ is positively invariant with respect to the closed loop system $\dot{x}=f(x)+g(x) \hat{k}(x)$.

\section{CONCLUSION}

This paper presents minimal barrier functions, which stem from scalar differential inequalities, to give the minimum assumptions for utilizing a continuously differentiable barrier function. We have characterized a class of comparison systems viable for verifying invariance of sets defined via a barrier function inequality and have proposed equivalent computable conditions. By formulating necessary and sufficient conditions for minimal barrier functions, the relation to Nagumo's theorem is also elucidated. We then directly extend minimal barrier functions to control formulations and propose relevant conditions for the existence of valid continuous controllers. By characterizing this relationship with the classical approach of verifying set invariance and rooting the proposed formulation directly in differential inequalities, this paper aims to provide a theoretical foundation for minimal barrier functions. Possible extensions include generalizing minimal barrier functions to hybrid systems.

\section{REFERENCES}

[1] F. Blanchini and S. Miani, Set-theoretic methods in control. Springer, 2008.

[2] A. D. Ames, J. W. Grizzle, and P. Tabuada, "Control barrier function based quadratic programs with application to adaptive cruise control," in 53rd IEEE Conference on Decision and Control, pp. 6271-6278, IEEE, 2014.

[3] A. D. Ames, X. Xu, J. W. Grizzle, and P. Tabuada, "Control barrier function based quadratic programs for safety critical systems," IEEE Transactions on Automatic Control, vol. 62, no. 8, pp. 3861-3876, 2017.

[4] M. Nagumo, "Über die lage der integralkurven gewöhnlicher differentialgleichungen," Proceedings of the Physico-Mathematical Society of Japan. 3rd Series, vol. 24, pp. 551-559, 1942.

[5] J.-M. Bony, "Principe du maximum, inégalité de harnack et unicité du probleme de cauchy pour les opérateurs elliptiques dégénérés," in Annales de l'institut Fourier, vol. 19, pp. 277-304, 1969.

[6] H. Brezis, "On a characterization of flow-invariant sets," Communications on Pure and Applied Mathematics, vol. 23, no. 2, pp. 261-263, 1970.

[7] G. Ladde, V. Lakshmikantham, et al., "On flow-invariant sets.," Pacific Journal of Mathematics, vol. 51, no. 1, pp. 215-220, 1974.

[8] R. Redheffer, "The theorems of bony and brezis on flow-invariant sets," The American Mathematical Monthly, vol. 79, no. 7, pp. 740-747, 1972.

[9] S. Prajna and A. Jadbabaie, "Safety verification of hybrid systems using barrier certificates," in International Workshop on Hybrid Systems: Computation and Control, pp. 477-492, Springer, 2004.

[10] S. Prajna, A. Jadbabaie, and G. J. Pappas, "A framework for worstcase and stochastic safety verification using barrier certificates," IEEE Transactions on Automatic Control, vol. 52, no. 8, pp. 1415-1428, 2007.

[11] R. Wisniewski and C. Sloth, "Converse barrier certificate theorems," IEEE Transactions on Automatic Control, vol. 61, no. 5, pp. 1356-1361, 2015.

[12] J.-P. Aubin, Viability theory. Springer Science \& Business Media, 2009.

[13] H. Kong, F. He, X. Song, W. N. Hung, and M. Gu, "Exponentialcondition-based barrier certificate generation for safety verification of hybrid systems," in International Conference on Computer Aided Verification, pp. 242-257, Springer, 2013.

[14] D. D. Bainov and P. S. Simeonov, Integral inequalities and applications, vol. 57. Springer Science \& Business Media, 2013.

[15] V. Lakshmikantham and S. Leela, Differential and Integral Inequalities: Theory and Applications: Volume I: Ordinary Differential Equations. Academic press, 1969.

[16] R. P. Agarwal and V. Lakshmikantham, Uniqueness and nonuniqueness criteria for ordinary differential equations, vol. 6. World Scientific Publishing Company, 1993.

[17] M. Maghenem and R. G. Sanfelice, "Characterizations of safety in hybrid inclusions via barrier functions," in Proceedings of the 22nd ACM International Conference on Hybrid Systems: Computation and Control, pp. 109-118, ACM, 2019.

[18] M. Maghenem and R. G. Sanfelice, "Sufficient conditions for forward invariance and contractivity in hybrid inclusions using barrier functions," arXiv preprint arXiv:1908.03980, 2019.

[19] R. M. Redheffer and W. Walter, "Flow-invariant sets and differential inequalities in normed spaces," Applicable Analysis, vol. 5, no. 2, pp. 149-161, 1975.

[20] A. D. Ames, S. Coogan, M. Egerstedt, G. Notomista, K. Sreenath, and P. Tabuada, "Control barrier functions: Theory and applications," arXiv preprint arXiv:1903.11199, 2019.

[21] E. Michael, "Continuous selections. i," Annals of mathematics, pp. 361$382,1956$.

[22] X. Xu, P. Tabuada, J. W. Grizzle, and A. D. Ames, "Robustness of control barrier functions for safety critical control," IFAC-PapersOnLine, vol. 48 , no. 27 , pp. 54-61, 2015.

[23] B. J. Morris, M. J. Powell, and A. D. Ames, "Continuity and smoothness properties of nonlinear optimization-based feedback controllers," in 2015 54th IEEE Conference on Decision and Control (CDC), pp. 151-158, IEEE, 2015.

[24] W. W. Hogan, "Point-to-set maps in mathematical programming," SIAM review, vol. 15, no. 3, pp. 591-603, 1973.

[25] C. Berge, Topological Spaces: including a treatment of multi-valued functions, vector spaces, and convexity. Courier Corporation, 1997. 